\title{
CIUDADANÍA TRANSNACIONAL Y DERECHO PENAL: A PROPÓSITO DEL CASO EUROPEO*
}

\author{
TRANSNATIONAL CITIZENSHIP AND CRIMINAL LAW: \\ ABOUT THE EUROPEAN CASE \\ CitOYENNETÉ TRASNATIONAL ET DROIT PÉNAL: \\ A PROPOS DEL CASE EUROPÉEN
}

Jonatan VAlenZuela Saldías ${ }^{* *}$

\begin{abstract}
RESUMEN
El marco politico de comprensión de la ciudadanía se ve intensamente puesto a prueba por los intentos de construcción de espacios de deliberación transnacionales. El caso de la denominada "Constitución Europea" resulta paradigmático a este respecto. Esta tensión tiene un aspecto de relevancia frente a la justificación filosófico politica del derecho penal: el reconocimiento y las expectativas politicas determinan la forma que el derecho penal ha de tener en el contexto de estos nuevos espacios de deliberación.
\end{abstract}

PalabRas Clave: Ciudadanía - Derecho - Derecho Penal - Constitución Europea

ABSTRACT

The political framework of citizenship is intensely tested by attempts to build transnational spaces of deliberation. The case of the so-called "European Constitution" is paradigmatic in this respect. The tension has an aspect of policy relevance from the perspective of philosophical justification of criminal law: recognition and political expectations determine how criminal law must be shaped in the context of these new spaces of deliberation.

KEY WORDS: Citizenship - Law - Criminal law - European Constitution

RÉSUME

Le cadre politique de connaissance de la citoyenneté est mise à l'épreuve par les tentatives de construire des espaces transnationaux de délibération. À cet égard, on peut trouver un exemple paradigmatique dans la "Constitution européenne". Cette tension est importante face à la justification philosophique du droit pénal: la reconnaissance et les attentes politiques modèlent la manière dans laquelle le droit pénal doit être compris dans le contexte des nouveaux espaces de déliberation.

MoTS CLÉs: Citoyenneté - Droit - Droit penal - Constitution européenne

* Artículo recibido el 30 de septiembre de 2013 y aceptado para su publicación el 15 de noviembre de 2013.

** Licenciado en Ciencias Jurídicas y Sociales por la Universidad de Chile. Doctor en Derecho por la Universidad de Girona. Investigador del Centro de Estudios de la Justicia de la Facultad de Derecho de la Universidad de Chile. Correo electrónico: jvalenzuela@derecho.uchile.cl. 


\section{INTRODUCCIÓN}

Los intentos de ampliación del marco político que desembocan en el proceso de integración regional tienen en la Unión Europea una experiencia de especial fuerza. La inclusión de un determinado país en el seno del grupo de países que integra una comunidad conlleva su participación en un intrincado proceso de adaptación a lo que puede llamarse "un espacio político común". Una parte importante de este proceso -la denominada Constitución Europea- expresa con particular énfasis este punto: ¿ha logrado cuajarse un acto de constitución jurídica del espacio político compartido en Europa?

Junto a este proceso, se sostiene que el Derecho tal y como lo conocemos está en crisis. Se dice que parte de esa crisis se expresa en la necesidad de revisión de las pretensiones de comprensión del lenguaje del Derecho estatal tradicional frente al lenguaje de un nuevo orden jurídico generado a partir de la idea de establecimiento de orden globalizado. La ley como mecanismo de expresión de la idea del pueblo es actualmente una herramienta débil, en cambio el contexto constitucional (y en muchas ocasiones meta-constitucional) y su reconocimiento de derechos cuyos contenidos pueden ser reclamados directamente por sus titulares constituye una solución más eficiente ${ }^{1}$. La ley parece haber sido desplazada por el contrato y por tanto lo que queda a los ciudadanos para relacionarse con la comunidad política debe ser algo así como un "espacio constitucional" en el que las herramientas con las que el ciudadano cuenta son, simplemente, derechos.

Esta situación impacta, entre otros, en la comprensión de Derecho Penal. Tradicionalmente se situaba la idea de punición frente a un estado nación que determinaba su contenido. La pregunta sobre qué es delito y qué debe ser penado públicamente se afirma en una relación casi exacta con la idea de comunidad nacional. Hoy en día, frente a intentos como el de la Constitución Europea ${ }^{2}$ y a la aparente crisis del derecho, aparece la necesidad de articular estas decisiones en un contexto transnacional o de, al menos, generar desde un contexto transnacional las decisiones de persecución penal. El estado nacional y la ley ya no aparecen como el terreno obvio en el que las decisiones de incriminación y persecución penal tienen lugar ${ }^{3}$.

En el presente trabajo se intenta reflexionar sobre la figura de ciudadanía presente en la denominada Constitución Europea y su correlato con la idea de ciudadano para la articulación de un argumento de justificación del derecho penal.

\footnotetext{
${ }^{1}$ El denominado lenguaje de los derechos frente a la crisis del lenguaje del derecho ha sido tratado, entre otros, por Ferrarese, Maria Rosaria (2002). Il diritto al presente: Globalizzazione e tempo delle istituzioni. Bologna: Il Mulino, pp. 135-185.

2 En adelante CE.

${ }^{3}$ En el caso chileno puede apreciarse claramente este punto a propósito de la generación en nuestro derecho de la Ley No 20.393 que establece un régimen de responsabilidad penal de las personas jurídicas. La idea de legislar y la ley en concreto fueron claramente determinadas por la necesidad que el Estado de Chile tenía de cumplir ciertos requisitos de ingreso a la OCDE.
} 
No se trata estrictamente de un trabajo sobre el sentido de la regulación que puede ser llamada CE, sino que, a propósito de este debate se sitúa a las intuiciones de justificación del Derecho Penal frente a este proceso.

Atendido lo anterior el presente trabajo tiene su base en las siguientes ideas:

(1) El concepto de ciudadano europeo tiene un carácter formal y prima facie insatisfactorio con una tesis de legitimación del Derecho Penal democráticamente comprometida;

(2) Ese carácter formal y teóricamente insatisfactorio tiende a su vinculación con el nivel constitucional diluyendo, potencialmente, el sentido de la ley;

(3) Ese recurso al nivel constitucional es un síntoma que lleva a la revisión del modelo democrático que se encuentra detrás del hipotético plan punitivo europeo;

(4) Esa revisión nos obliga a poner sobre la mesa qué clase de tesis legitimatoria puede tenerse en el contexto penal asumiendo un compromiso democrático con esa operación.

\section{El CiUdAdAnO EUROPEO}

Europa vive un dilema: el reconocimiento. Con la aproximación de la lógica política del resultado se han producido una serie de reflexiones que tienen como resultado a la denominada CE. En lo que nos interesa, el nervio central de esta declaración está dado por la idea de ciudadano europeo que aparece expresada del siguiente modo:

"Artículo I-10. Ciudadanía de la Unión

1. Toda persona que tenga la nacionalidad de un Estado miembro posee la ciudadanía de la Unión, que se añade a la ciudadanía nacional sin sustituirla.

2. Los ciudadanos de la Unión son titulares de los derechos y están sujetos a los deberes establecidos en la Constitución. Tienen el derecho:

a) de circular y residir libremente en el territorio de los Estados miembros;

b) de sufragio activo y pasivo en las elecciones al Parlamento Europeo y en las elecciones municipales del Estado miembro en el que residan, en las mismas condiciones que los nacionales de dicho Estado;

c) de acogerse, en el territorio de un tercer país en el que no esté representado el Estado miembro del que sean nacionales, a la protección de las autoridades diplomáticas y consulares de cualquier Estado miembro en las mismas condiciones que los nacionales de dicho Estado. d) de formular peticiones al Parlamento Europeo, de recurrir al Defensor del Pueblo Europeo, así como de dirigirse a las instituciones y a los órganos consultivos de la Unión en una de las lenguas de la Constitución y de recibir una contestación en esa misma lengua.

3. Estos derechos se ejercerán en las condiciones y dentro de los limites definidos por la Constitución y por las medidas adoptadas en aplicación de ésta". 
Como puede apreciarse, la ciudadanía europea contemplada en la CE depende del reconocimiento de la ciudadanía de alguno de los países miembros de la Unión Europea ${ }^{4}$. Las cosas aparecerían sencillas en la medida que se considere a esta metaciudadanía como una manera de ampliar el acceso a derechos de los sujetos al régimen político europeo.

Sin embargo, la ciudadanía frente a la articulación de un programa de punición no sólo tiene esta dimensión. El reconocimiento de ciudadanía de un sujeto implica la posibilidad de actuar políticamente y por ello recibir el reproche expresado en la ley de la comunidad en la que vive. El ciudadano es por definición autor de la comunidad en la que vive, es quien tiene en su mano (al menos en abstracto) la posibilidad de cambiar o mantener un determinado plan político5.

¿Puede la ciudadanía europea modificar esta circunstancia? Si consideramos que la ciudadanía europea tiende al entendimiento de la UE como una "comunidad política" se crea un conflicto claro. ¿Cuál es la comunidad a la que pertenece el ciudadano europeo? ¿Quiénes le reconocen como un igual? ¿Qué plan punitivo le pertenece?

La ciudadanía necesita situarse en un plan democráticamente satisfactorio y ese plan pasa por el reconocimiento de los miembros de la comunidad. Esta es una cuestión básica en el Derecho Penal actual, la existencia de un autor en términos mínimamente igualitarios permite la elaboración del predicado del delito respecto de una acción que le pertenece. Es necesario, entonces, que podamos atribuir un contenido material a la idea de ciudadanía que sea operativa para el armado del juicio de imputación.

A pesar de esto, en el nivel de la ciudadanía europea no existe un nivel legal que, tal como en el contexto de los estados nacionales, permita reconocer un mecanismo de representación de las ideas que cada ciudadano pueda tener respecto de las decisiones públicas que afectarán su vida. Es más, la idea del penado cada vez más se asocia a un sujeto "distinto" del ciudadano común y corriente cobrando con ello mucha fuerza la necesidad de mayor intervención y protección de la comunidad general ${ }^{6}$.

La ciudadanía -frente al Derecho- debe ser capaz de expresar agencia. Esta agencia no se encuentra desprovista de contenido moral. Que uno pertenezca o sea suscriptor de un acuerdo político, naturalmente, requiere una sección material. La responsabilidad como idea "preconcebida" o actitud prima facie otorga sentido a la ciudadanía ${ }^{7}$.

\footnotetext{
${ }^{4}$ En adelante UE.

${ }^{5}$ Vid. Mañalich Raffo, Juan Pablo (2005). "Pena y ciudadanía”. Revista de Estudios de la Justicia, No 7 , pp. 63-83.

${ }^{6} \mathrm{La}$ posición del acto es una cuestión controvertida. Para una reconstrucción de la responsabilidad criminal sin necesidad de acto vid. HusaK, Douglas (1998). "Does Criminal Liability requires an act?". En: Philosophy and the criminal law. Principle and Critique, Duff, R.A. (Ed.), Cambridge: University Press, pp. 60-100.

${ }^{7}$ La responsabilidad o el merecimiento derivado de la noción de responsabilidad ha sido tomado, entre otros, por Moore. Para una lectura interesante frente a la tensión con una aproximación dialéctica sobre
} 
Estimo que en los contornos de la determinación del ciudadano europeo, se niega espacio para que la intuición moral sobre la responsabilidad sea operativa y más aún, ciertamente legítima. La pérdida de la deliberación democrática que puede apreciarse al vincular el reconocimiento material al nivel nacional genera una carencia de sentido de la idea de ciudadano, con lo que, en realidad, el argumento de montaje de un plan punitivo europeo en este contexto es tan sólo una intuición y no un modelo jurídico que reconozca una intuición.

\section{La lógica del Derecho frente a la lógica de los Derechos}

En la Filosofía del Derecho se ha enfrentado desde hace algún tiempo la denominada lógica del derecho con la denominada lógica de los derechos ${ }^{8}$.

La lógica del derecho se encuentra referida al sistema de normas establecidas en un contexto estatal y que derivan del ejercicio de la soberanía. La configuración del plan político de una comunidad en esta lógica, se expresa paradigmáticamente en la ley.

Se ha dicho que la ley es un mecanismo, en algún sentido, poco dinámico pero que debiera expresar lo que los integrantes de una determinada comunidad quieren respecto de su plan de vida en común.

Se sostiene que el Derecho (y con ello se alude a la ley como forma de expresión del derecho) se encuentra en problemas en tanto creación jurídica exhaustiva9 El lenguaje propio de la ley se ha sustituido -en el contexto de la debilitación del estado nacional- por el lenguaje del contrato.

El contrato como forma dinámica de razonamiento jurídico ha superado, se sostiene, a la ley como expresión inmóvil del acuerdo jurídico ${ }^{10}$. El contrato entendido como un acuerdo intersubjetivo flexible que permite a las partes llegar a acuerdos conforme a sus particulares intereses deviene en una forma de regulación de las relaciones sociales tanto más efectiva que la ley. Hoy en día, las grandes transnacionales definen, en los hechos, el contenido de las relaciones laborales en diversos países sin que la expresión legal pueda contener a la rapidez y flexibilidad de la lógica contractual.

Esta lógica se asocia a la idea de derechos. Los derechos tienden a una relación sincrónica en el tiempo, permiten su modificación y derogación debido a que dependen en su operatividad de su invocación coyuntural para la configuración de un acuerdo contingente. El derecho por su parte es diacrónico, expresa un

el individuo frente al derecho vid. Norrie, Alan (2000). Punishment, Responsability and Justice. Oxford: Clarendon Press, pp. 95-116.

${ }^{8}$ Vid., entre otros, Ferrarese (2002), pp. 135-185.

9 Ferrarese (2002), 135.

${ }^{10}$ Ferrarese (2002), p. 136. 
acuerdo en un momento determinado y con ello "fija" la decisión independiente de su invocación coyuntural ${ }^{11}$.

Además, la lógica de los derechos y del contrato pone su atención en las prestaciones de que disponen los sujetos desde el reconocimiento de cierto espacio de intangibilidad de intereses. La lógica de los derechos puede prescindir del nivel legal pues se permite la solución del reconocimiento con el nivel constitucional. Justamente es en esta lógica en la que se inscribe el intento de esta meta-ciudadanía europea.

El modelo de gobierno al cual se vincula al "ciudadano europeo" es un modelo donde la deliberación democrática se encuentra en segundo plano. Lo que importa es la instancia de solución de controversias con base en el mayor cúmulo de derechos que pueden invocarse.

Si la controversia se produce respecto a la decisión misma entonces el ciudadano tiene que apelar a la empatía política con aquellos que decidan en el contexto de la UE, pero no puede oponerse en términos serios, porque se encuentra teóricamente vedado su acceso al mecanismo de legitimación legal ${ }^{12}$.

Se cree que allí donde se establezca una entrada al goce de ciertos derechos, entonces cada ciudadano puede ir y buscar lo que se piensa es un correlato obvio de ese goce: el mecanismo de aplicación directa de ese derecho ${ }^{13}$.

Esta es una tesis que tiende a vincular a los sujetos con su (pretendido) contenido de derechos y a mirar la generación legal como un obstáculo para el goce completo de esos derechos. Por ello, incluso se ha hablado de la necesidad de concebir un "constitucionalismo global"14.

Esta es la solución menos democrática que podríamos asociar a la idea de ciudadano europeo frente al Derecho Penal. Si existe necesidad de vincular al ciudadano (y su deliberación) a un contexto propio de la lógica del constitucionalismo (global) tenemos que la idea de individuo y de su deliberación se torna irrelevante. Se piensa que los derechos, que en apariencia, aportan prestaciones a sus titulares, pueden suspender la expresión constitutiva de los mismos ${ }^{15}$.

\footnotetext{
${ }^{11}$ Ferrarese (2002), p. 140.

${ }^{12}$ Y el modo por definición para la solución de conflictos serán los denominados "ADR", o mecanismos alternativos de resolución de conflictos, que también obedecen a la lógica contractual. Si nos detenemos en este escenario veremos que las formas de solución de conflictos aún en el proceso penal se acercan a este modo.

${ }^{13}$ Sobre el escenario de los derechos (humanos) y su aplicación vid. Atria Lemaitre, Fernando (2003). "Ubi Ius, Ibi Remedium? La relevancia jurídica de los derechos humanos". Revista de Estudios de la Justicia, No 3, pp. 35-47.

14 Vid. Ferrajoli, Luigi (1998). "Más allá de las soberanía y la ciudadanía: un constitucionalismo global”. En: Isonomía, No 9, pp. 173-184.

${ }^{15}$ Esta es, además, una tesis abiertamente contramayoritaria. Tanto la idea de constitucionalismo global como de sustitución del lenguaje del derecho por el de los derechos entrañan considerar como un detalle dentro del concepto de convivencia democrática a la idea de mayoría. Bien podríamos abandonar a la mayoría en la
} 


\section{Democracia deliberativa y Derecho Penal}

El Derecho Penal es probablemente una de las ramas del Derecho que más intensamente reflejan la identidad de una comunidad.

Una reivindicación central para el ejercicio de la punición es la valoración de la participación de los ciudadanos en la discusión pública que tiende al impacto en el modelamiento del sistema penal ${ }^{16}$. Esta participación de los ciudadanos debe desembocar en una propiedad relevante del sistema: su legitimidad.

Tanto desde la perspectiva utilitarista como desde la perspectiva retributiva se ha llegado a un consenso relativo a que la práctica punitiva de una determinada comunidad necesita de un espacio institucional de deliberación que robustezca el concepto de democracia ${ }^{17}$. La existencia de deliberación democrática configura, entonces, a las reglas para la práctica punitiva.

¿Existe deliberación democrática en el contexto de la ciudadanía europea? La respuesta es negativa. La negativa puede expresarse en tres pasos:

El primer paso es el de la ciudadanía "base" o ciudadanía nacional. En la medida que se opte por la preferencia de la ley como expresión de las guías del modelo de persecución penal esta ciudadanía nacional es suficiente para legitimar el sistema nacional. Si por el contrario se prefiere a las "razones constitucionales" que dan lugar a la lógica de los derechos, entonces la ciudadanía base o nacional carece de sentido. Si no podemos determinar el destino del plan político de nuestra comunidad nuestra deliberación deviene en tautología.

El hecho de poder informarse y realizar un acto de expresión de reproche o apoyo a una determinada versión del uso del poder en nuestras comunidades constituye por una parte a la comunidad jurídica en la que vivimos y por otro nos constituye precisamente como integrantes de la comunidad.

El segundo paso se refiere a los mecanismos de representación. ¿Es posible que una ciudadanía no exprese el reconocimiento necesario para dar legitimidad? En algún sentido es posible por medio de la disolución de la representación directa en los actores políticos. La ética de los acuerdos de la clase política como cuestiones que se anteponen a las decisiones del ciudadano redunda en falta de participación y con ello en exclusión.

Así, la clase que ejerce el poder selecciona a quienes ratificarán su versión de ejercicio del poder, excluyendo a los que carecen de una "fe" suficiente para montar un plan alternativo.

medida que aquellos que puedan determinar el contenido de los derechos (en el nivel político) se entiendan en un lenguaje común con los llamados a aplicar ese contenido (el juez). Esta es una tesis errónea en varios sentidos. Considero, sin embargo, que no es este el lugar para extenderme sobre el punto.

${ }^{16}$ En este sentido vid. Dzur, Albert y Mirchandani, Rekha (2007). "Punishment and democracy. The role of public deliberation”. En: Punishment \& Society, No 9, pp. 151-175.

17 Desde la perspectiva utilitarista y republicana vale la pena ver BraithwaIte, John y PetTit, Philip (1990). Not just Desert: A Republican theory of criminal justice. Oxford: Oxford University Press. 
El tercer paso, es el de la transnacionalización de la ciudadanía. Desde luego podemos advertir que la existencia de una ciudadanía que exceda los límites del estado nacional es vista como un fenómeno que porta progresos en materia de protección de derechos fundamentales. Sin embargo, el hecho de la transnacionalización importa una disolución de las propiedades que, desde la óptica del Derecho Penal, son necesarias para comprender un determinado plan punitivo como legítimo ${ }^{18}$.

Podemos ver que la toma de decisiones al interior de la UE está desprovista de participación directa en las soluciones y de hecho no se enfrenta al ciudadano de manera directa, en tanto considera ciudadanos europeos a sujetos que tengan calidad de ciudadano reconocido por los subsistemas democráticos que integran a la UE.

El Derecho Penal constituye un sistema de imputación, es decir un sistema de reglas que tienden al señalamiento y adjudicación de las consecuencias de determinada acción designada a su vez como delito. Para poder ser imputado el autor debe ser reconocido como miembro de una comunidad política. El autor debe ser considerado un igual en los términos del juego de reconocimiento recíproco que constituye a la comunidad en la que realiza el hecho delictivo ${ }^{19}$.

Por ello la pregunta sobre la ciudadanía europea es la pregunta por el reconocimiento, por la pertenencia a una determinada comunidad de los denominados ciudadanos europeos. Tan sólo aquellos que pueden ser imputados -reconocidos entonces- pueden encontrarse en la obligación de tolerar legítimamente las penas que puedan imponerse en el contexto de este acuerdo político transnacional.

Una vía de justificación está determinada por considerar dos espacios de democracia para legitimar un sistema penal. En este sentido puede considerarse que existe un plano de democracia cosmopolita y otro de democracia local.

El plano cosmopolita incluye los acuerdos democráticos locales que tienden a un fin de mantención de la paz común y se asocia entonces a un "meta-plan" que contiene las necesidades de punición de los demás planes políticos.

Este es un intento de fundamentación para los planes punitivos diseñados para traspasar el contexto del estado nacional ${ }^{20}$.

La cuestión a la que se enfrenta centralmente esta justificación es a la correlación con las decisiones del nivel constitucional interno de cada uno de las comunidades que integran el "meta plan".

\footnotetext{
${ }^{18}$ Debería considerarse, en algún sentido, lo dispuesto en torno al sufragio para el parlamento europeo. Si ese mecanismo ocupara un espacio relevante dentro del espacio de deliberación democrática de los países miembros de la UE, entonces podríamos responder dudosamente la pregunta por la deliberación en el espacio de acción de la ciudadanía europea.

${ }^{19}$ En un sentido similar vid. Mañalich RafFo (2005), pp. 64-65.

${ }^{20}$ Vid. Ralph, Jason (2003). "Between Cosmopolitan and American Democracy: Understanding US Opposition to the International Criminal Court". En: International Relations, No 17, pp. 195 y ss.
} 
Esto es natural, el acuerdo democrático que pertenece a los ciudadanos es aquel en el que pueden intervenir, es aquel con el que se relacionan como autores. Las demás decisiones le son ajenas.

¿Puede la comunidad sin más aceptar entonces la estructuración de un plan de persecución política transnacional?

Sin perjuicio de los problemas generales de mecánica de justificación de la ciudadanía como fundamento de la práctica punitiva dentro de una comunidad, probablemente el mayor de los problemas se aprecia en torno al reconocimiento de los que forman la propia comunidad y de quienes son "otros".

Offe ha puesto de manifiesto esta cuestión. El modelo político de la UE tropieza con la identificación de quiénes son los ciudadanos y quiénes son los otros $^{21}$. Respecto de los ciudadanos podemos tener una razonable expectativa de comportamiento y sin embargo no todos los sujetos que quedan comprendidos dentro de la expresión ciudadano europeo son reconocidos como iguales por otros sujetos. Así ha dicho:

"Quizás se haya agotado el repertorio de valores y principios con los que se fundamentaba 'Europa'. El examen del estado de la argumentación normativa con la que fundamentar el proyecto de una Unión Europea política, si bien no lleva a resultados totalmente negativos, sí resultan al menos ambivalentes. Al mismo tiempo, parece crecer con el avance de la integración la necesidad de motivaciones que sirvan a los ciudadanos europeos para identificarse con el proyecto de integración política independientemente de los intereses nacionales o de grupo" 22 .

Si consideramos el contexto normativo desde el que puede reflexionarse al "ciudadano europeo" tenemos que el obstáculo que ve Offe es natural. Si la pertenencia a la comunidad política europea descansa en la pertenencia -efectiva- a una ciudadanía nacional, entonces en realidad el contexto es una tensión entre distintos planes políticos que pueden tender a desconocerse materialmente y a no encontrar obstáculo en la instrumentalización del otro.

A modo de conclusión

La decisión que en materia política se asuma para comprender un determinado campo del derecho tiene la mayor relevancia. Soy consciente de que muchos de los argumentos presentados requieren una explicación (las más de las veces larga) sobre el punto de partida.

Como hemos visto, un sistema político igualitario necesita al Derecho para sujetarse, ya no bajo la idea (despótica) ilustrada, sino para sujetarse en tanto Constitución. El nivel constitucional es precisamente el punto sobre el que debe descansar un sistema legítimo, por ello el nivel constitucional no puede ser usado

${ }^{21}$ Offe, Claus (2000). "Democracia y Estado de Bienestar: un régimen europeo bajo la tensión de la integración europea”. En: Zona Abierta, No 92-93, pp. 243-282.

22 Offe (2000), p. 279. 
como razón para obviar el nivel legal. La Constitución es una ley fundamental, pero es una ley. Y los ciudadanos son, en un sentido esencial, autores de sus leyes.

Esta es la manera en que la lectura crítica que ofrece Offe puede tener más sentido. En realidad los intentos de integración política en Europa descansan sobre una ilusión cómoda: que los europeos se reconocen entre sí como iguales.

Un punto de partida, para el caso del Derecho Penal, es, a mi juicio, la reconsideración de la función que un plan punitivo tiene o debería tener dentro de una comunidad. Salvando el absurdo de la tesis utilitaria, podemos comprender el sentido de nuestros espacios de castigo y con ello, coherentemente, administrar los argumentos para construir una instancia de punición que desborde los límites nacionales pero no los límites de legitimidad otorgados por la idea de ciudadanía.

La ciudadanía europea entonces, tiene un carácter meramente formal. Ratifica el hecho de la existencia de la vinculación entre un sujeto y una comunidad política nacional (la ciudadanía base) pero no es capaz de expresar una vinculación política de relevancia con miras a la legitimación de una determinada práctica punitiva.

Esa ineptitud de la ciudadanía europea, ha permitido que se generen en torno a la idea de transnacionalización y globalización argumentos de sustitución del reconocimiento de la ley como reflejo de una vinculación política efectiva. El denominado neo-constitucionalismo ha encontrado en la falta de capacidad expresiva de estos planes, un contexto donde desarrollar el argumento incorrecto de evaluar positivamente el lenguaje de los derechos y sus consecuencias para los titulares.

Este intento, de otra parte, permite revisar el valor que un modelo democrático nacional puede tener frente a la articulación de un determinado derecho penal. La idea de deliberación sólo puede tener sentido en la medida que sea una acción correlativa a la vida política de unos sujetos determinados en un contexto aprehensible.

Ante ello, el Derecho Penal se enfrenta a la pregunta sobre su sentido. Una práctica punitiva con base en una teoría retributiva permite (como en otros contextos) resistir de manera fiable la horadación a la idea de democracia que puede encontrarse tras las consecuencias de esta forma de articulación de la ciudadanía transnacional. En este punto una teoría preventiva o utilitaria es esencialmente estéril.

Pero este no es el único punto que debe ser considerado. La justificación en el nivel filosófico político del Derecho Penal debe incluir, sobre todo en el caso del Derecho Penal, aquellos acuerdos acerca de lo que rechazamos en la vida en comunidad. Deberíamos enfrentar un escenario en que pueda ponerse de manifiesto qué clase de tesis acerca de la democracia se sostenga como correcta y subsecuentemente enfrentar a una zona del Derecho que descansa esencialmente en la administración de males y castigos.

Parte de la respuesta pasa por asumir algún contexto de justificación política del Derecho de manera transparente. No son pocos los intentos por situar al Derecho 
Penal encajado en la actual discusión de filosofía política. En este sentido, vale la pena tener presente que la lectura que tradicionalmente se asume en el ámbito de la justificación del Derecho Penal es en algún sentido tributaria de la denominada perspectiva liberal.

Esta no es la única perspectiva disponible. Desde el punto de vista de la defensa de una noción fuerte de democracia, la tesis republicana ha cobrado cierta relevancia en esta clase de tópicos.

Es complejo dar una noción clara de republicanismo en el Derecho y aún más en el Derecho Penal. Besson y Martí han intentado dar un grupo de nociones que parecieran ser compartidas por los diversos autores que se inscriben en este marco de pensamiento. Al respecto sostienen que el republicanismo implica una defensa de la noción de libertad que rompe con el tradicional marco berliniano de libertad positiva y negativa, postulando un ideal de libertad como no dominación, en la que se asumen la presencia de interferencias pero se proscribe a las interferencias arbitrarias y sobre todo a los casos de dominación (aún sin interferencia) ${ }^{23}$.

Además se sostiene una noción fuerte de igualdad, considerada precondición de la libertad. Probablemente una de las consecuencias más intensas desde el punto de vista de la ciudadanía y el discurso de legitimación del Derecho Penal sea la idea de autogobierno y democracia que una teoría republicana del Derecho Penal pueda contener.

Desde esta perspectiva, se ha defendido intensamente un ideal de democracia deliberativa, que pone en primer plano la noción de deliberación como mecanismo racional de adopción de decisiones en la vida pública ${ }^{24}$.

Este mecanismo supone cierta fenomenología en torno al espacio en que se delibera y sobre quienes pueden participar de manera relevante en la deliberación. Es por ello que puede entenderse en la perspectiva republicana que los ciudadanos pueden y deben ser considerados autores del plan punitivo.

En el marco de la ampliación transnacional del ejercicio del ius puniendi, precisamente este ideal queda en entredicho. Esto puede conducirnos a una consecuencia de extrema relevancia: el Derecho Penal en el contexto de la negación de la deliberación democrática puede volverse una herramienta de la tiranía. Con ello, poco queda del ideal ilustrado de un Derecho Penal justificado democráticamente, pues justamente la legitimación democrática deliberativa puede devenir en irrelevante.

${ }^{23}$ Besson, Samantha y Martí, José Luis (2009). "Law and Republicanism: Mapping the Issues”. En: Legal Republicanism. National and International Perspectives, Besson, Samantha y MARTí, José Luis (Eds.), Oxford: Oxford University Press, pp. 3-36.

${ }^{24}$ Fundamental en este aspecto resulta MARTí, José Luis (2006). La República deliberativa. Una teoría de la democracia. Barcelona: Marcial Pons, pp. 24 y ss. 


\section{BiBLIOGRAFÍA CITADA}

Atria Lemaitre, Fernando (2003). "Ubi Ius, Ibi Remedium? La relevancia jurídica de los derechos humanos". Revista de Estudios de la Justicia, No 3, pp. 35-47.

Besson, Samantha y MarTí, José Luis (2009). "Law and Republicanism: Mapping the Issues". En: Legal Republicanism. National and International Perspectives, Besson, Samantha y MarTí, José Luis (Eds.), Oxford: Oxford University Press, pp. 3-36.

Braithwaite, John y Pettit, Philip (1990). Not just Desert: A Republican theory of criminal justice. Oxford: Oxford University Press.

Dzur, Albert y Mirchandani, Rekha (2007). "Punishment and democracy. The role of public deliberation”. En: Punishment \& Society, No 9, pp. 151-175.

Ferrajoli, Luigi (1998). "Más allá de las soberanía y la ciudadanía: un constitucionalismo global”. En: Isonomía, No 9, pp. 173-184.

FERRARESE, Maria Rosaria (2002). Il diritto al presente: Globalizzazione e tempo delle istituzioni. Bologna: Il Mulino.

HusaK, Douglas (1998). “Does Criminal Liability requires an act?”. En: Philosophy and the criminal law. Principle and Critique, Duff, R.A. (Ed.), Cambridge: University Press, pp. 60-100.

Karstedt, Susanne y Lafree, Gary (2006). "Democracy, Crime and Justice". En: The Annals of the American Academy of Political and Social Science, No 605, pp. 6-23.

Mañalich Raffo, Juan Pablo (2005). "Pena y ciudadanía”. Revista de Estudios de la Justicia, No 7, pp. 63-83.

Mañalich Raffo, Juan Pablo (2007). "La pena como retribución”. Revista de Estudios Públicos, No 108, pp. 117-205.

MarTí, José Luis (2006). La República deliberativa. Una teoría de la democracia. Barcelona: Marcial Pons.

Moore, Michael (1997). Placing Blame. A General Theory of the Criminal Law. Oxford: Clarendon Press.

Norrie, Alan (2000). Punishment, Responsability and Justice. Oxford: Clarendon Press.

Offe, Claus (2000). "Democracia y Estado de Bienestar: un régimen europeo bajo la tensión de la integración europea”. En: Zona Abierta, No 92-93, pp. 243282.

RalPH, Jason (2003). "Between Cosmopolitan and American Democracy: Understanding US Opposition to the International Criminal Court". En: International Relations, No 17. 